\title{
ТЕПЛООБМЕН ПРИ ФИЛЬТРАЦИИ ЖИДКОСТИ В КОЛЬЦЕВОМ КАНАЛЕ, ЗАПОЛНЕННОМ ПОРИСТОЙ СРЕДОЙ
}

\author{
Дехтярь Руслан Анатольевич, \\ dekhtyar@itp.nsc.ru
}

\author{
Институт теплофризики СО РАН, \\ Новосибирск, Россия, 630090, г. Новосибирск, пр. Академика Лаврентьева, 1.
}

\begin{abstract}
Актуальность работы обусловлена широким использованием пористых сред как при проектировании и оптимизации компактных теплообменников, так и в инженерных расчётах характеристик тепломассообмена в каналах с зернистой средой и при обтекании тел, погруженных в пористую среду.

Цель: экспериментальное исследование процесса переноса тепла от стенки кольцевого канала к фильтрующейся через пористую вставку жидкости при различных режимах течения; получение полуэмпирических корреляций для теплообмена в инерционном режиме фильтрации жидкости в кольцевом канале.
\end{abstract}

объект: кольцевой канал с пористой вставкой, состоящей из стеклянных шариков одного диаметра с разными типами как регулярных, так и хаотических упаковок.

Методы: экспериментальные методы поиска закономерностей процессов теплообмена. В опытах с помощью термопар измерялась температура обогреваемой внешней стенки кольцевого канала в четырех сечениях и температура нагревателя 8 двух разных точках. Также измерялись температуры на входе в рабочий участок и на выходе из него. Одновременно с тепловыми исследованиями измерялся расход жидкости при фрильтрации через пористую вставку. Пористость определялась обычным весовым способом. В специальных тарировочных опытах отдельно определялись тепловые потери рабочего участка. Расчет коэффрициента теплоотдачи проводился по подводимому потоку тепла к наружной стенке кольцевого канала с учетом тепловых потерь и по разности измеряемой температуры стенки и среднемассовой температуры жидкости в данном сечении.

Результаты. Представлены результаты экспериментального исследования теплообмена фильтрационного потока жидкости со стенками кольцевого канала, заполненного пористой средой, при постоянном тепловом потоке на внешней стенке. Исследование проводилось на кольцевых каналах с различной шириной. По ширине канала укладывался или один слой шаров одного диаметра с двумя типами упаковок: кубической и ромбоэдрической, или несколько слоев шаров, но уже с произвольной упаковкой (от 3 до 10 шаров по ширине канала). При обработке экспериментальных данных в качестве определяющего геометрического параметра выбран гидравлический диаметр зерна. Показано, что в зависимости от режима фильтрации через пористую вставку существуют различные законы теплообмена. Так, для турбулентного режима фильтрации полученные данные для теплообмена хорошо обобщаются универсальным «законом двух третей». Приведено сравнение с данными других авторов для теплообмена в кольцевом канале и в круглой трубе. Показано, что в инерционном режиме фильтрации теплообмен соответствует закономерности Nu Re ${ }^{1 / 2}$.

\section{Ключевые слова:}

Фильтрация, теплообмен, пористая среда, кольцевой канал, гидравлический диаметр.

\section{Введение}

Изучение процессов передачи тепла при вынужденной конвекции через пористые среды является одним из самых сложных фундаментальных проблем теплофизики. Необходимость изучения и исследования процессов тепломассопереноса в пористых средах связана с важными инженерными и промышленными приложениями. В этих приложениях решается ряд таких проблем, как интенсификация добычи нефти и газа методами теплового воздействия на продуктивные пласты $[1,2]$, сбор и удаление разлитой нефти, с фильтрацией и гидрогеологическим моделированием, а также эксплуатация геотермальных источников теплоты. Физическое и численное моделирование применяется как для построения теоретических моделей фильтрации, так и для решения различных задач, в том числе: оценки запасов подземных вод, расчета понижений уровня подземных вод при подтоплении, водоотборе, при создании водохранилищ или от работ оросительных систем [3]. Несомненную ценность имеют эти исследования для геодинамики, а именно для исследования тектоники плит, которая является поверхностным проявлением тепловой конвекции, происходящей главным образом благодаря действию радиоактивных источников теплоты и охлаждению Земли. Кроме того, не менее важными являются задачи, связанные с сооружением подземных хранилищ, прокладкой теплотрасс и других коммуникаций, в том числе в условиях вечной мерзлоты [4]. Еще одно очень важное приложение, в котором использование пористой среды нашло свое применение при проектировании и совершенствовании компактных и эффективных теплообменников, например, с концентрическими трубами [5], - это традиционная энергетика. В ядерной энергетике широкое применение получили реакторы с шаровыми тепловыделяющими элементами [6-8].

Анализ процессов конвективного переноса в каналах, заполненных пористой средой, существенно усложняется. Это связано с тем, что пористая среда является весьма непростым объектом для исследования. Например, при обтекании отдельных элементов пористой вставки возникают вихревые и струйные течения, усложняющие анализ картины течения или фильтрации жидкости через пористую среду. Существует некоторая неопределенность при выборе ха- 
рактерных размеров, а также отмечается резкое различие, например, между теплопроводностью твердого каркаса, составляющего основу пористой среды, и жидкостью, фильтрующейся через эту среду. Это значительно затрудняет обработку опытных данных, поэтому вводится понятие эффективных коэффициентов переноса [9]. Как показали исследования, стенка канала оказывает упорядывающее воздействие на плотность упаковки зерен в пористой вставке, которая изменяется от 1 на стенке до примерно 0,4 в ядре для хаотической упаковки. Изменение пористости в зависимости от расстояния от стенки приводит к появлению «канального эффекта», то есть к локальному максимуму скорости вблизи стенки [10-13].

Для описания теплообмена в пористых средах в теоретических и расчетных моделях используются традиционные представления о ламинарном и турбулентном пограничных слоях. Полагают, что перенос теплоты происходит за счет смешения струй жидкости при обтекании элементов пористого слоя. Чаще всего рассматривается двухслойная схема процесса, где отдельно выделены пристенная область с линейным профилем температур и шириной не более одного диаметра шарика $d$ и ядро засыпки, где предполагается, что скорость фильтрации постоянна [9]. В самом простом случае при анализе течения в поровом или межзерновом пространстве используется закон сопротивления Дарси:

$$
\frac{\partial p}{\partial x}=\frac{\mu}{K} u
$$

где $p$ - давление жидкости в пористой среде, Па; $\mu$ динамическая вязкость, Па·с; $K$ - проницаемость пористой среды, м²; $u$ - средняя скорость жидкости; $x$ вдоль оси, м/с.

При моделировании теплообмена в пористой среде в работах $[14,15]$ показано, что использование закона Дарси в виде (1) в сочетании с ударным профилем скорости при малых скоростях фильтрации дает хорошие результаты.

В более сложных моделях закон сопротивления Дарси в виде (1) модифицируют, пытаясь учесть вязкое трение на твердых стенках, ограничивающих пористую среду, изменение пористости около стенки, а также инерционные потери давления при высоких скоростях [11, 13, 15-18].

При экспериментальном исследовании теплообмена в пористой среде усилия исследователей чаще всего направлены на получение универсальных зависимостей, которые были бы справедливы в широком диапазоне режимных параметров [12, 19-21]. Методика обработки опытных данных сводится к получению эмпирической зависимости для коэффициента теплоотдачи, зависящего от режимных и геометрических параметров, а также от теплофизических свойств жидкости:

$$
\mathrm{Nu}=c \cdot f(d / D) \cdot \operatorname{Re}^{n} \operatorname{Pr}^{m},
$$

где $f$ - функция, зависящая от геометрических параметров пористого слоя; $d$ - диаметр шариков, из которых состоит пористая среда; $D$ - диаметр рабочего канала; показатели степени $n$ и $m$ подбираются из эксперимента. Из анализа большого массива экспериментальных данных разных авторов следует, что показатель $n$ для сопоставимых коэффициентов теплопроводности твердой $\lambda_{S}$ и жидкой $\lambda_{L}$ фазы может изменяется в разных опытах от 0,6 до 1,0 [22]. Что же касается показателя $m$ в формуле (2), то и тут нет особой ясности. Так, из анализа опытных данных работ разных авторов следует, что величина $m$ может изменяется от $1 / 3$ до 2/3. При расчете по формуле (2) и обработке опытных данных существует неопределенность при выборе в качестве характерного масштаба длины при вычислении безразмерных параметров $\mathrm{Nu}$ и $\mathrm{Re}$ - это может быть диаметр трубы $D$, диаметр элемента пористой среды $d$ или эквивалентный (гидравлический) диаметр $d_{e}$

Следует отметить еще то, что для рассматриваемой в настоящей работе теплоотдачи в кольцевом канале в зависимости от режима фильтрации существуют различные законы теплоотдачи к стенке трубы по аналогии с круглой трубой $[23,24]$. При увеличении числа Рейнольдса изменяется не только картина течения жидкости, но при двухслойном характере теплопереноса происходит перераспределение вкладов ядра потока и пристенной зоны в суммарное термическое сопротивление $[4,23,25]$. При этом необходимо учитывать то обстоятельство, что пристенная зона характеризуется упорядоченной или регулярной упаковкой, а также повышенной пористостью.

Таким образом, исследование теплоотдачи в кольцевом канале с возможностью варьирования количества шаров в поперечном сечении кольцевого канала вплоть до одного является наиболее привлекательным и удобным способом для выяснения влияния роли ядра потока и пристенной зоны.

\section{Методика эксперимента}

На рис. 1 приведена схема экспериментального стенда, представляющего собой замкнутый циркуляционный контур. Рабочая жидкость с помощью центробежного насоса -5 из бака - 4 подавалась в рабочий участок - 1 . После прохождения рабочего участка жидкость поступала в расходомерный участок - 2, охлаждалась в кожухотрубном теплообменнике -3 и возвращалась в бак - 4. Для поддержания постоянного расхода через пористую среду при малых скоростях фильтрации использовалась байпасная линия -6 . Расход жидкости варьировался от 0,007 до 0,4 кг/с.

На рис. 2 приведена схема рабочего участка, который был изготовлен из медной цилиндрической трубы -1 внутреннего диаметра $D_{2}=52$ мм с толщиной стенки 1,6 мм. На наружную стенку трубы была наклеена слюда в два слоя, а уже на нее намотан проволочный нихромовый электрический нагреватель - 4 , который был подключен к источнику постоянного тока. С помощью этого источника регулировалась мощность нагревателя и количество подведённого тепла к стеке трубы. Чтобы уменьшить тепловые потери в окружающую среду электронагреватель был изолирован слоем тепловой изоляции - 6. Для создания кольцевого канала внутрь медной трубы поме- 
щался специально изготовленный центральный вытеснитель - 2 из тонкостенной стеклянной трубки, заполненной воздухом и запаянной с обеих сторон. Конфигурация вытеснителя имела хорошо обтекаемые формы. Таким образом, на внешней стенке центрального вытеснителя выполнялось условие адиабатичности, а на внешней стенке медной трубы - постоянство теплового потока. Длина рабочего участка составила 131 мм, а длина обогреваемого участка 125 мм.

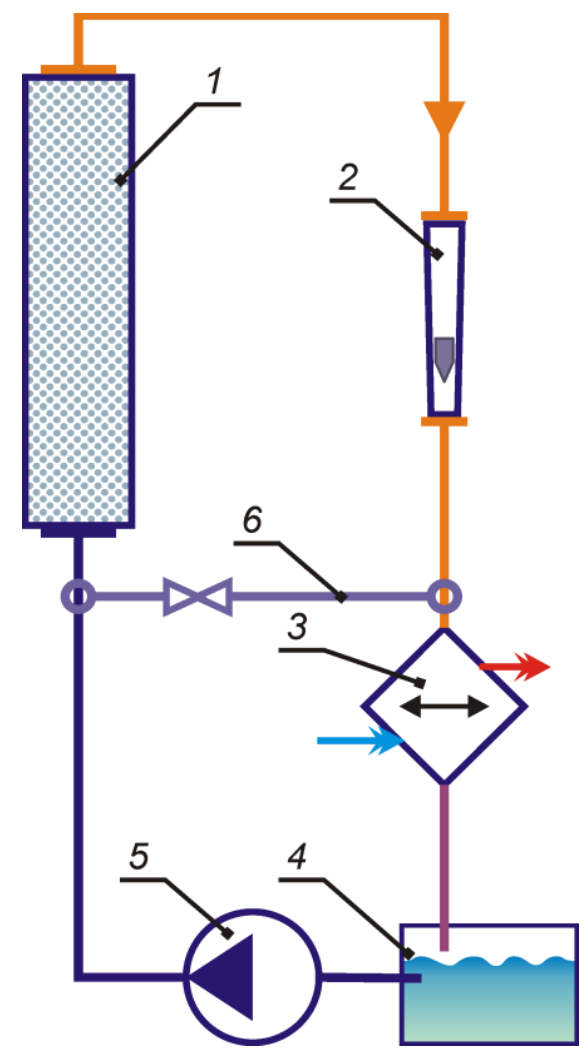

Pис. 1. Схема установки: 1 - рабочий участок; 2 - расходомерный участок; 3 - теплообменник; 4 бак; 5 -насос; 6-байпасная линия

Fig. 1. Schematic of the experimental installation: 1 working section; 2 -flow-meter section; 3 - heat exchanger; 4 -tank; 5 -pump; 6-bypass line

Кольцевой канал заполнялся стеклянными шариками диаметром $d=8,9$ мм. При этом был выбран наружный диаметр центрального вытеснителя $D_{1}=34$ мм с тем, чтобы по ширине кольцевого канала мог поместиться только один шарик. В таком канале были реализованы два типа регулярных упаковок: кубическая и ромбоэдрическая. Таким образом, была сформирована однослойная упаковка шариков с заданными шириной канала и диаметром шариков.

Кольцевой канал также заполнялся стеклянными шариками диаметром $d=3,2$ и 0,9 мм. При этом был выбран наружный диаметр центрального вытеснителя $D_{1}=30$ мм для $d=3,2$ мм и $D_{1}=42$ мм для $d=0,9$ мм с тем, чтобы по ширине кольцевого канала помещалось от 3 до 10 шариков соответственно. При такой укладке шаров в кольцевом канале была реализована хаотическая или нерегулярная упаковка.

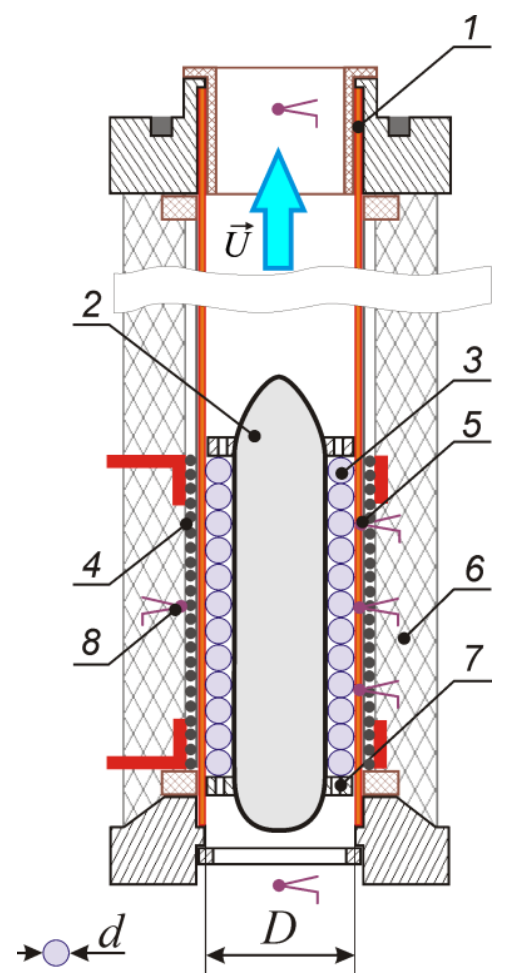

Pис. 2. Схема рабочего участка: 1 - медная труба; 2 иентральный вытеснитель; 3 - пористая среда; 4 - нагреватель; 5 - медно-константановые термопары; 6 - слой теплоизоляции; 7 - решётка; 8 - нихром-константановые термопары

Fig. 2. Diagram of the working section: 1 - copper pipe; 2 - central displacer; 3 - porous medium; 4 -heater, 5 - copper-constantan thermocouples; 6 - heat insulation layer; 7 - grid; 8) nichrome-constantan thermocouples

Для регулярных и хаотических упаковок шариков весовым способом была измерена пористость $\varepsilon$. В результате измерений было получено, что для шариков диаметром $d=8,9$ мм пористость $\varepsilon=0,47$ (кубическая упаковка) и $\varepsilon=0,41$ (ромбоэдрическая упаковка); для шариков диаметром $d=3,2$ мм пористость $\varepsilon=0,36$; для шариков диаметром $d=0,9$ мм пористость $\varepsilon=0,38$.

После заполнения канала шариками - 3 (рис. 2) пористый слой жестко фиксировался решетками - 7, которые были установлены как на входе, так и на выходе из рабочего участка.

С помощью четырех медно-константановых термопар - 5 с диаметром проволок 0,18 и 0,1 мм, соответственно, измерялась температура стенки трубы. Нихром-константановыми термопарами - 8 с диаметром проволок 0,1 мм в двух разных точках измерялась температура нагревателя. Такими же термопарами измерялись температуры на входе в рабочий участок и на выходе из него. После обогреваемого участка с пористой вставкой был установлен смесительный участок, состоящий из четырёх медных дисков с отверстиями, разделенных кольцевыми прокладками. Это устройство обеспечивало равномерное перемешивание нагретой рабочей жидкости после выхода из обогреваемого рабочего участка. Контроль за качеством работы смесителя осуществлялся при помощи 
двух нихром-константановых термопар с диаметром проволок 0,1 мм. Термопары располагались в одном поперечном сечении: одна термопара - точно на оси трубы, а другая - на расстоянии 5 мм от стенки трубы Опыты показали, что разность показаний термопар, расположенных после смесителя, не превышала $0,1^{\circ} \mathrm{C}$, что свидетельствовало о том, что степень перемешивания жидкости таким простым устройством была очень эффективной. Все используемые в экспериментах термопары были откалиброваны с точностью в пределах $\pm 0,1{ }^{\circ} \mathrm{C}$.

В специальных тарировочных опытах определялись тепловые потери с наружной поверхности рабочего участка. Коэффициент теплоотдачи рассчитывался по подводимому потоку тепла к наружной стенке кольцевого канала за вычетом теплопотерь и по разности измеряемой температуры стенки и среднекалориметрической температуры рабочей среды в данном сечении.

Одновременно с термопарными измерениями измерялся расход жидкости, фильтрующейся через пористую вставку в кольцевом канале. В качестве рабочей жидкости использовалась дистиллированная вода

\section{Результаты и обсуждения}

Исследовался теплообмен при течении рабочей жидкости (воды) в кольцевом канале с шаровой засыпкой из стеклянных шариков с теплопроводностью, близкой к теплопроводности воды для граничного условия $q_{w}=$ const. Обсуждаемые ниже результаты относятся к стабилизированному участку теплообмена при постоянном тепловом потоке на внешней стенке и отсутствии отвода тепла на внутренней стенке кольцевого канала.

Эксперименты проводились в кольцевом канале, заполненном пористой средой, как с регулярной упаковкой (кубической и ромбоэдрической), состоящей из одного слоя шаров диаметром $d=8,9$ мм, так и с нерегулярной упаковкой, состоящей из нескольких слоев шаров, помещающихся в кольцевом зазоре (с шариками диаметра $d=3,2$ и 0,9 мм).

На рис. 3 представлены опытные данные для кольцевого канала, заполненного шариками с $d=8,9$ мм (кубическая и ромбоэдрическая упаковки), 3,2 и 0,9 мм (хаотическая упаковка). Из рисунка видно, что в турбулентном режиме фильтрации $\left(\operatorname{Re}_{e}>70\right)$ данные по теплообмену в кольцевом канале с однослойной кубической и ромбоэдрической упаковкой шаров с $d=8,9$ мм хорошо согласуются между собой. Экспериментальные результаты полностью соответствуют универсальному «закону степени 2/3», на который было указано в работах $[23,24]$. В этих же работах было показано, что в зависимости от режима фильтрации через пористую вставку существуют различные законы теплообмена. В результате исследований было выделено три режима фильтрации: инерционный, переходный и турбулентный.

В терминах эквивалентных характерных масштабов этот универсальный закон может быть записан в следующем виде:

$$
\mathrm{Nu}_{e}=0,27 \cdot \operatorname{Re}_{e}^{2 / 3} \cdot \operatorname{Pr}^{0,4}
$$

где $\mathrm{Nu}_{e}=\alpha \cdot d_{e} / \lambda_{L}, \operatorname{Re}_{e}=U \cdot d_{e} /(\varepsilon v), U=u \varepsilon-$ истинная скорость,

$$
d_{e}=d \cdot \frac{\varepsilon}{1,5 \cdot(1-\varepsilon)+d / D}
$$

где $d_{e}$ - гидравлический диаметр, который подсчитан с учетом площади стенок, для кольцевого канала $D=D_{2}-D_{1}$, а для круглой трубы $D=D_{2}$. Для трубы с большим количеством шаров по сечению $d_{e}$ обычно рассчитывают по формуле:

$$
d_{e}=\frac{2}{3} \cdot \frac{\varepsilon}{1-\varepsilon} \cdot d
$$

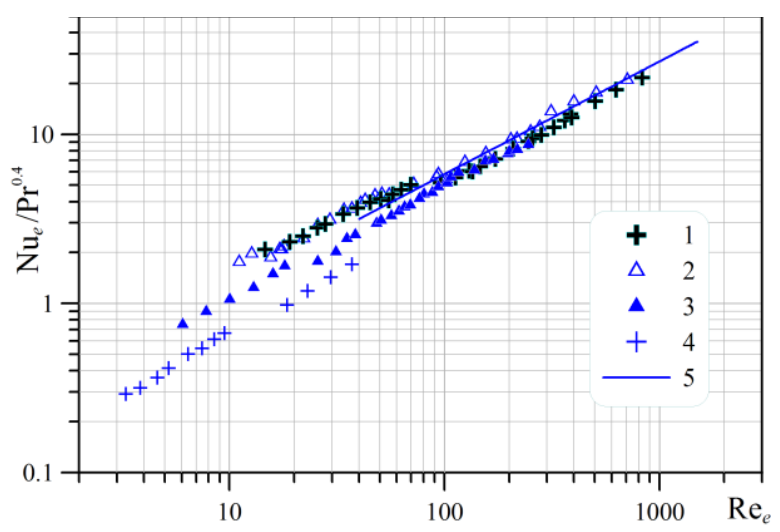

Pис. 3. Теплоотдача в кольцевом канале: 1) $d=8,9$ мм, $D_{l}=34$ мм (кубическая упаковка); 2) $d=8,9$ мм, $D_{I}=34$ мм (ромбоэдрическая упаковка); 3 ) $d=3,2 \mathrm{Mм}$, $D_{I}=30$ мм (хаотическая упаковка); 4) $d=0,9$ мм, $D_{I}=42$ мм (хаотическая упаковка); 5) эмпирическая зависимость $\mathrm{Nu}_{e}=0,27 \mathrm{Re}_{e}{ }^{2 / 3} \mathrm{Pr}^{0,4}$

Fig. 3. Heat transfer in the annular channel: 1) $d=8,9 \mathrm{~mm}$, $D_{l}=34 \mathrm{~mm}$ (cubic packing); 2) $d=8,9 \mathrm{~mm}, D_{I}=34 \mathrm{~mm}$ (rhombohedral packing); 3) $d=3,2 \mathrm{~mm}, D_{l}=30 \mathrm{~mm}$ (random packing); 4) $d=0,9 \mathrm{~mm}, D_{l}=42 \mathrm{~mm}$ (random packing); 5) empirical dependence $\mathrm{Nu}_{e}=0,27 \operatorname{Re}_{e}^{2 / 3} \operatorname{Pr}^{0,4}$

Из рис. 3 также видно, что при турбулентной фильтрации $\left(\operatorname{Re}_{e}>70\right)$ в кольцевом канале через пористую вставку с шариками $d=3,2$ мм (хаотическая упаковка шаров) также реализуется универсальный «закон двух третей» для теплоотдачи. Но как видно из рисунка, экспериментальные точки на графике лежат примерно на $10 \%$ ниже эмпирической зависимости (3) - сплошная линия 5. Можно добиться совпадения экспериментальных точек с эмпирической зависимостью, записанной в виде (3), если принять во внимание то, что по сечению канала укладывалось не более трех шаров, а при пересчете чисел $\mathrm{Nu}$ и $\mathrm{Re}$ вместо скорости фильтрации взять истинную скорость, соответствующую кубической упаковке.

На рис. 4 представлены результаты измерения безразмерного коэффициента теплоотдачи как в кольцевом канале, так и в круглой трубе с пористой вставкой в зависимости от числа Рейнольдса [23]. Приведено сопоставление экспериментальных данных, полученных при фильтрации воды через стеклян- 
ные шарики, помещённые в кольцевой канал, с данными работы [26]. В качестве характерного масштаба длины, обобщающего данные и для трубы, и для кольцевого канала, выбран гидравлический диаметр, представленный по формуле (4) и характеризующий размер межзернового пространства. Из анализа результатов, представленных на этом рисунке следует, что при турбулентной фильтрации жидкости через пористые вставки в трубе и кольцевом канале теплообмен соответствует универсальному закону (3). Из рис. 4 хорошо видно, что можно выделить диапазоны чисел Рейнольдса, при которых экспериментальные данные по теплоотдаче, как в круглом, так и в кольцевом канале с одним слоем шаров регулярной и хаотической упаковок, могут быть аппроксимированы закономерностью вида: $\mathrm{Nu}_{e} \sim \operatorname{Re}_{e}^{1 / 2}$ (на рис. 4 - пунктирные линии 9).

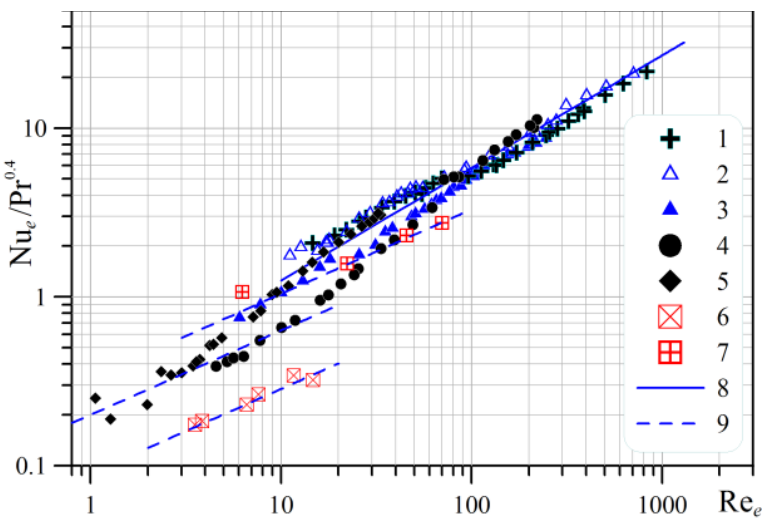

Puc. 4. Теплоотдача в кольиевом канале $(1-3,6,7)$ и круглой трубе (4, 5): 1) $d=8,9$ мм (кубическая упаковка); 2) $d=8,9$ мм (ромбоэдрическая упаковка); 3) $d=3,2 \mathrm{Mм}$ (хаотическая упаковка), данные настоящей работы; 4, 5) $d=3,2 \mathrm{Mм}(д а н-$ ные из [23] для фильтрации воды и $47 \%$ раствора глицерина, $D_{2}=52 \mathrm{Mм}$ ); 6, 7) $d=0,7$ и 2,5 мм (данные из [26], $\left.D_{2}=28 \mathrm{Mм}, D_{l}=9,5 \mathrm{Mм}\right) ; 8$ ) эмпирическая зависимость $\mathrm{Nu}_{e}=0,27 \mathrm{Re}_{e}^{2 / 3} \mathrm{Pr}^{0,4}$; 9) $з$ зкономерность $\mathrm{Nu}_{e} \sim \mathrm{Re}_{e}{ }^{1 / 2}$

Puc. 4. Heat transfer in the annular channel $(1-3,6,7)$ and in the round tube $(4,5): 1) d=8,9 \mathrm{~mm}$ (cubic packing), 2) $d=8,9 \mathrm{~mm}$ (rhombohedral packing); 3) $d=3,2 \mathrm{~mm}$ (random packing), data of this work; 4, 5) $d=3,2 \mathrm{~mm}$ (data of work [23] to filter water and $47 \%$ glycerin solution); 6, 7) data of work [26], $d=0,7 \mathrm{~mm}, d=2,5 \mathrm{~mm}$, respectively, $\left.D_{1}=9,5 \mathrm{~mm}, D_{2}=28 \mathrm{~mm} ; 8\right)$ empirical dependence $N u_{e}=0,27 \mathrm{Re}_{e}^{2 / 3} \mathrm{Pr}^{0,4}$; 9) regularity $N u_{e} \sim \operatorname{Re}_{e}{ }^{1 / 2}$

Как видно из рисунка, экспериментальные данные в области инерционного режима фильтрации при такой обработке расслаиваются. Поэтому для обобщения данных в области чисел Рейнольдса $\mathrm{Re}_{e}<70$, соответствующих инерционному режиму фильтрации, предлагается эмпирическая зависимость в следующем виде:

$$
\mathrm{Nu}_{e}=C \cdot\left(d_{e} / D\right) \cdot \operatorname{Re}_{e}^{1 / 2} \cdot \operatorname{Pr}^{1 / 2},
$$

где $C$ - константа эксперимента.

На рис. 5, 6 представлены основные результаты обработки опытных данных для общего коэффициен- та теплоотдачи на стабилизированном участке теплообмена в кольцевом канале и в круглой трубе.

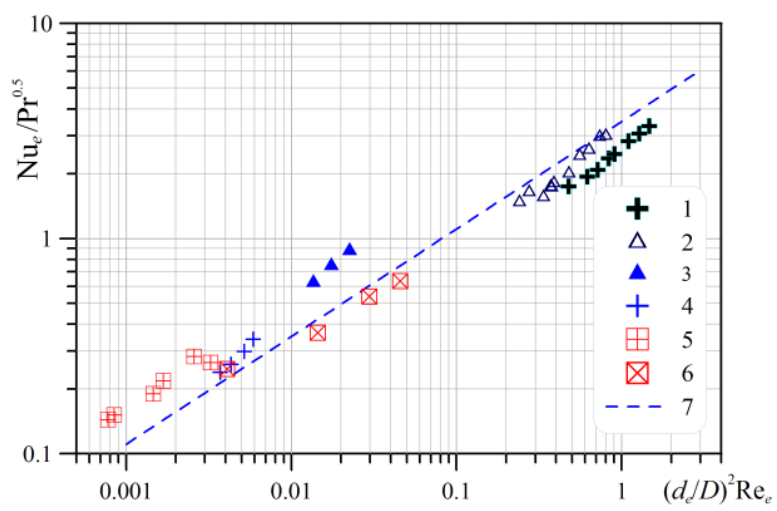

Рис. 5. Теплоотдача в кольцевом канале, соответствующая инерционному режиму течения жидкости. 1) $d=8,9$ мм (кубическая упаковка); 2) $d=8,9 \mathrm{Mм}$ (ромбоэдрическая упаковка); 3) $d=3,2$ мм (хаотическая упаковка); 4) $d=0,9$ мм (хаотическая упаковка), данные настоящей работыл; 5) $d=0,7$ мм; 6) $d=2,5$ мм (данные из работьл [26], $D_{l}=9,5$ мм, $D_{2}=28 . м$ ); 7) закономерность $N u_{e} \sim R e_{e}{ }^{1 / 2}$

Fig. 5. Heat transfer in the annual channel corresponding to the inertial regime of fluid flow: 1) cubic packaging, $d=8,9 \mathrm{~mm}$; 2) rhombohedral packaging, $d=8,9 \mathrm{~mm}$; 3) chaotic packaging, $d=3,2 \mathrm{~mm}$; 4) chaotic packaging, $d=0,9 \mathrm{~mm}$ (data of this work); 5 , 6) data of work [26] $d=0,7 \mathrm{~mm}, d=2,5 \mathrm{~mm}\left(D_{l}=9,5\right.$ $\left.\mathrm{mm}, \mathrm{D}_{2}=28 \mathrm{~mm}\right) ; 7$ ) regularity $N u_{e} \sim \mathrm{Re}_{e}{ }^{1 / 2}$

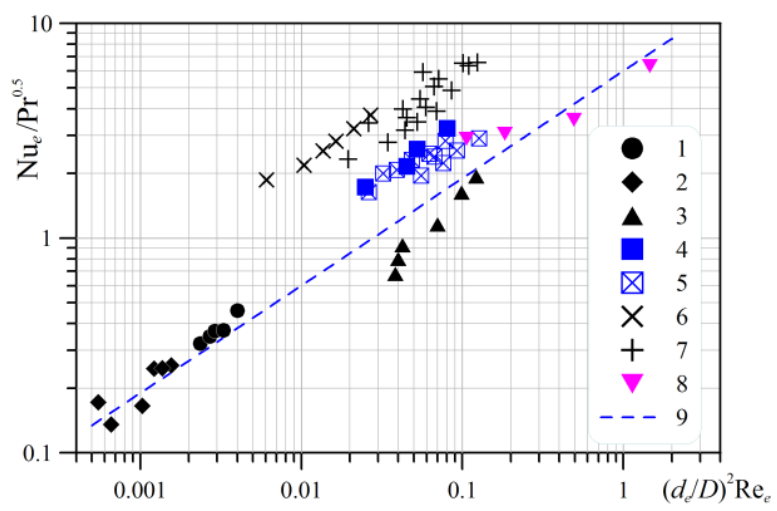

Pис. 6. Теплоотдача в круглой трубе при инериионном режиме фильтрации жидкости: 1) $d / D=0,062$ (вода); 2) $d / D=0,062$ (47 \% раствор глицерина); 3) $d / D=0,17$ (вода, данные из работы [23]); 4) $d / D=0,07$ (воздуx); 5) $d / D=0,11$ (воздух, данные из работь [19]); 6) $d / D=0,04 \quad$ (воздуx); 7) $d / D=0,07$ (воздух, данные из работы [27]); 8) $d / D=0,098$ (вода, данные из работы [287); 9) закономерность $N u_{e} \sim \operatorname{Re}_{e}{ }^{1 / 2}$

Fig. 6. Heat exchange in a pipe with inertial filtration of liquid: 1) $d / D=0,062$ (water); 2) $d / D=0,062$ (47\% glycerol solution); 3) $d / D=0,17$ (water, data from the work of [23]); 4) $d / D=0,07$ (air); 5) $d / D=0,11$ (air, data from the work of [19]); 6) $d / D=0,04$ (air); 7) $d / D=0,07$ (air, data from the work of [27]); 8) $d / D=0,098$ (water, data from the work of [28]); 9) regularity $N u_{e} \sim R e_{e}{ }^{1 / 2}$ 
Из рисунков видно, что при инерционном режиме фильтрации жидкости экспериментальные данные разных авторов хорошо соответствуют зависимости вида (5).

Рассматриваемую область чисел Рейнольдса квалифицируют как соответствующую инерционному режиму фильтрации, тем самым подчеркивая преобладающую роль инерционных сил и указывая на нелинейный характер закона сопротивления [23]. В этой области роль ядра потока становится преобладающей, а механизм процессов теплопереноса принципиальным образом отличается от механизма теплообмена в области турбулентной фильтрации.

Анализ опытных данных других авторов показывает, что результаты измерений среднего коэффициента теплоотдачи могут различаться в разы $[22,29]$, что связано, скорее всего, с погрешностями в измерениях температуры рабочей жидкости на выходе из пористой вставки, а также с использованием длинных рабочих каналов. Из-за малых разностей измеряемой температуры стенки и температуры жидкости происходит резкое увеличение погрешностей измеряемых и вычисляемых величин. Следует также иметь в виду, что при малых числах Рейнольдса $\mathrm{Re}_{e}$ и умеренных числах Прандтля Pr коэффициент теплопроводности каркаса пористой среды становится существенной величиной. Поэтому методически бывает трудно сделать оценку вклада пристенной зоны в общий коэффициент теплоотдачи.

Известно, что при массообмене сопротивление переносу вещества сосредоточено в основном у стенки канала из-за высоких значений числа Шмидта Sc. Поэтому в области невысоких чисел Рейнольдса $\mathrm{Re}_{e}$, coответствующих инерционному режиму течения, peзультаты измерения пристенного коэффициента массопереноса на стенке канала с пористой средой наиболее надежные и достоверные. Например, авторами работы [30] была предложена следующая эмпирическая корреляция:

$$
\mathrm{Nu}_{e}=A \cdot \operatorname{Re}_{e}^{1 / 2} \cdot \mathrm{Sc}^{1 / 3},
$$

справедливая в узком диапазоне чисел Рейнольдса $\operatorname{Re}_{e}=1 \ldots 40$, где константа пропорциональности $A$ зависит от условий проведения экспериментов и геометрических параметров. В таком случае можно производить расчет теплообмена по зависимостям, полученным из экспериментов по массообмену. Тогда необходимо принимать во внимание то обстоятельство, что при расчете не будут учтены некоторые

\section{СПИСОК ЛИТЕРАТУРЫ}

1. Мусакаев Н.Г., Бородин С.Л. Расчет термодинамических параметров опускного течения теплоносителя в скважине с учетом протаивания многолетнемерзлых пород // Известия Томского политехнического университета. Инжиниринг георесурсов. - 2020. - Т. 331. - № 3. - С. 135-144.

2. Сохошко С.К. Учет изменения закона фильтрации при расчете дебита газовой скважины // Известия Томского политехнического университета. Инжиниринг георесурсов. - 2020. Т. 331. - № 12. - С. 77-83.

3. Исследование процессов фильтрации воды в пористой среде методами физического и численного моделирования важные факторы, влияющие на итоговую величину коэффициента теплоотдачи: дополнительное термическое сопротивление контакта шариков со стенками трубы, теплопроводность шаровой засыпки и другие факторы. Следовательно, такие расчеты могут привести к несколько заниженным значениям коэффициента теплоотдачи.

Сопоставление эмпирической корреляции, записанной в виде (6) и полученной для пристенного тепломассообмена, с закономерностями, следующими из результатов исследования теплоотдачи в кольцевом канале для диапазона чисел Рейнольдса $\mathrm{Re}_{e}$, предшествующих турбулентной фильтрации в кольцевом канале и в трубе (рис. 5, 6), позволяет сделать вывод об аналогии процессов переноса как в пристенной зоне, так и в ядре потока.

Таким образом, любая оценка вклада пристенной зоны в суммарный теплообмен в пористой среде, проведенная с использованием эмпирических корреляций, будет всегда ограниченной. Это связанно с тем, что существуют ограничения в выборе рабочих жидкостей, условий проведения экспериментов и так далее. Поэтому необходимо проведение дополнительных детальных исследований, направленных на выявление роли ядра потока и пристенной зоны при рассмотрении двухслойной схемы процесса, чтобы иметь более определенное представление об основных механизмах переноса тепла в пористых и зернистых средах.

\section{Заключение}

На основании проведенного исследования можно сделать следующие выводы:

1) теплоотдача от поверхности трубы, заполненной зернистым слоем, в режиме турбулентной фильтрации определяется закономерностями, присущими пристенной зоне, и подчиняется универсальному «закону двух третей» в форме (3);

2) в инерционном режиме фильтрации характер процессов переноса в ядре и в пристенной зоне одинаков, теплоотдача подчиняется закономерности $\mathrm{Nu}_{\mathrm{e}} \sim \operatorname{Re}_{\mathrm{e}}{ }^{1 / 2}$

3) необходимы детальные экспериментальные исследования, чтобы установить критерии, определяющие смену режимов теплообмена, характеризующие геометрию задачи, влияние физических свойств и параметров течения.

Работа выполнена в рамках проекта по госзаданию № 121031800213-0.

К.В. Белов, А.Б. Лисенков, А.Д. Пономарёв, Н.С. Горбатенко // Известия Томского политехнического университета. Инжиниринг георесурсов. - 2017. - Т. 328. - № 8. - С. 64-74.

4. Halkarni S.S., Sridharan A., Prabhu S.V. Experimental investigation on effect of random packing with uniform sized spheres inside concentric tube heat exchangers on heat transfer coefficient and using water as working medium // International Journal of Thermal Sciences. - 2018. - V. 133. - P. 341-356.

5. Проблемы контроля фильтрации вод через гидротехнические сооружения в условиях вечной мерзлоты / Н.В. Юркевич, Н.В. Юркевич, В.Н. Гуреев, Н.А. Мазов // Известия Томского политехнического университета. Инжиниринг георесурсов. 2020. - T. 331. - № 4. - C. 126-138. 
6. Богоявленский Р.Г. Гидродинамика и теплообмен в высокотемпературных ядерных реакторах с шаровыми твэлами. - М.: Атомиздат, 1978. - $112 \mathrm{c}$.

7. Сорокин В.В. Гидравлика и теплообмен шаровых засыпок в условиях активной зоны водо-водяных ядерных реакторов с микротвэлами. - Минск: Беларус. навука, 2010. - 193 с.

8. Экспериментальное исследование и численное моделирование гидродинамики и теплообмена в шаровых засыпках / А.В. Бороздин, А.Н. Варава, А.В. Дедов, А.Т. Комов, С.А. Малаховский, Ю.В. Сморчкова // Тепловые процессы в технике. 2015. - T. 7. - № 7. - С. 295-300.

9. Экспериментальное исследование теплопереноса в шаровой засыпке / А.А. Авдеев, Б.Ф. Балунов, Ю.Б. Зудин, Р.А. Рыбин $/ /$ Теплофизика высоких температур. - 2009. - Т. 47. - № 5. C. 724-733.

10. Lerou J.J., Froment G.F. Velocity, temperature and conversion profiles in fixed bed catalytic reactors // Chemical Engineering Science. - 1977. - V. 32. - Iss. 8. - P. 853-861.

11. Vafai K. Convective flow and heat transfer in variable-porosity media // Journal of Fluid Mechanics. - 1984 - V. 147. - P. 233-259.

12. Poulikakos D., Renken K. Forced convection in a channel filled with porous medium, including the effects of flow inertia, variable porosity, and Brinkman friction // Journal of Heat Transfer. 1987. - V. 109. - P. 880-888.

13. Гольдштик М.А. Процессы переноса в зернистом слое. 2-е изд., перераб. и доп. / отв. ред. Н.И. Яворский. - Новосибирск: Изд-во Института теплофизики СО РАН, 2005. - 358 с.

14. Li C.-H., Finlayson B.A. Heat transfer in packed beds - a reevaluation // Chemical Engineering Science. - 1977. - V. 32 (9). P. 1055-1066.

15. Vafai K., Tien C.L. Boundary and inertia effects on flow and heat transfer in porous media // International Journal of Heat and Mass Transfer. - 1981. - V. 24. - Iss. 2. - P. 195-203.

16. Lee D.Y., Vafai K. Analytical characterization and conceptual assessment of solid and fluid temperature differentials in porous media // International Journal of Heat and Mass Transfer. 1999. - V. 42. - P. 423-435.

17. Теплицкий Ю.С. О теплообмене в трубе, заполненной зернистым слоем // Инженерно-физический журнал. - 2004. T. 77. - № 1. - С. 86-92.

18. Сираев Р.Р. Фильтрация жидкости в пористой среде Форцгеймера с пространственно неоднородными пористостью и проницаемостью // Вычислительная механика сплошных сред. - 2019. - Т. 12. - № 3. - С. 281-292.

19. Yagi S., Kunii D. Studies on heat transfer in packed beds // International Development in Heat Transfer. - 1961. - Pt. IV. P. 750-759.

20. Storck A., Coeuret F. Mass transfer between a flowing liquid and a wall or an immersed surface in fixed and fluidized beds // Chemical Engineering Journal. - 1980. - V. 20. - P. 149-156.

21. Kays W.M., London A.L. Compact heat exchangers. - New York: McGraw Hill, 1984. -352 p.

22. Wakao N., Kaguei S., Funazkri T. Effect of fluid dispersion coefficients on particle-to-fluid heat transfer coefficients in packed beds: correlation of Nusselt numbers // Chemical Engineering Science. - 1979. - V. 34 (3). - P. 325-336.

23. Теплообмен в зернистом слое при умеренных числах Рейнольдса / Р.А. Дехтярь, Д.Ф. Сиковский, А.В. Горин, В.А. Мухин // Теплофизика высоких температур. - 2002. - Т. 40. № 5. - C. 748-755.

24. Dekhtyar R.A., Ovchinnikov V.V. An experimental study of hydrodynamics and heat transfer at fluid filtration through a porous medium // Journal of Physics: Conference Series. - 2019. 1359. -012122 .

25. Melanson M.M., Dixon A.G. Solid conduction in low dt/dp beds of spheres, pellets and rings // International Journal of Heat and Mass Transfer. - 1985. - V. 28. - P. 383-394.

26. Wang B-X., Du J-H. Forced convection heat transfer in a vertical annulus filled with porous media // International Journal of Heat and Mass Transfer. - 1993. - V. 36. - № 17. - P. 4207-4213.

27. Verschoor H., Schuit G.C.A. Heat transfer to fluids flowing through a bed of granular solids // Applied Scientific Research. 1951. - A2. - P. 97-119.

28. Nilles M., Martin H. Heat transfer at the wall of packed beds // Proc. 9th International Heat Transfer Conference. - New York: Hemisphere Publishing, 1990. - Р. 255-260.

29. Аэров М.Е., Тодес О.М. Гидравлические и тепловые основы работы аппаратов со стационарным зернистым слоем. - Л.: Химия, 1968. - 510 c.

30. Yagi S., Wakao N. Heat and mass transfer from wall to fluid in packed beds // American Institute of Chemical Engineering Journal. - 1959. - V. 5. - № 1. - P. 79-85.

Поступила 02.08.2021 2.

\section{Информация об авторах}

Дехтярь Р.А., кандидат технических наук, старший научный сотрудник, Институт теплофизики СО РАН. 
DK 532.546

\title{
HEAT TRANSFER DURING LIQUID FILTRATION IN AN ANNULAR CHANNEL FILLED WITH POROUS MEDIUM
}

\author{
Ruslan A. Dekhtyar, \\ dekhtyar@itp.nsc.ru
}

\author{
S.S. Kutateladze Institute of Thermophysics SB RAS, \\ 1, Academician Lavrentiev avenue, Novosibirsk, 630090, Russia.
}

\begin{abstract}
The relevance of the work is caused by the wide use of porous media, both in the design and optimization of compact heat exchangers and in the engineering calculations of the characteristics of heat and mass transfer in the channels with a grainy medium and when flowing around the bodies immersed in the porous medium.

The main aim of the research is the experimental study of heat transfer in an annular channel filled with a granular medium at various modes of liquid filtration; to obtain semi-empirical correlations for heat transfer in the inertial mode of fluid filtration in an annular channel. Objects: annular channel with a porous insert consisting of glass beads of the same diameter with different types of both regular and chaotic pakings.

Methods: experimental methods for finding the patterns of heat exchange processes. In experiments, using thermocouples, the temperature of the heated outer wall of the annular channel was measured in four sections and the temperature of the heater at two different points. The temperatures at the inlet to the working station and at the output of it were measured as well. Simultaneously with thermal investigation, the fluid flow was measured when filtering through a porous insert. Porosity was determined by the usual weighty way. In special calibration experiments, the heat losses of the working section were determined separately. The calculation of the heat transfer coefficient was carried out according to the flow of heat to the outer wall of the annular channel, taking into account heat losses and, along the difference in the measured wall temperature and the medium-mass fluid temperature in this section.

The paper introduces the results of the experimental study of liquid filtration flow heat exchange with the walls of the annular channel filled with a porous medium at constant heat flux on the outer wall. The study was conducted on ring channels with different widths. The width of the channel was laid either one layer of the balls of one diameter with two types of packages: cubic and rhombohedral, or several layers of balls, but already with arbitrary packaging (from 3 to 10 ball width). When processing the experimental data, hydraulic grain diameter is selected as the determining geometric parameter. It is shown that, depending on the filtration mode, various laws of heat exchange exist through a porous insert. So for the turbulent filtering mode, the obtained data for heat exchange is well summarized by the universal «law of two-thirds». The paper introduces the comparison with the data of other authors, both for heat exchange in the annular channel and in a

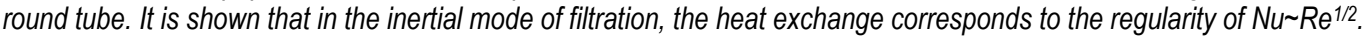

\section{Key words:}

Filtration, heat exchange, porous media, annular channel, hydraulic diameter.

The research was carried out within the project of the State Task no. 121031800213-0.

\section{REFERENCES}

1. Musakaev N.G., Borodin S.L. Calculation of thermodynamic parameters of the heat transfer agent downward flow in a well, taking into account permafrost melting. Bulletin of the Tomsk Polytechnic University. Geo Assets Engineering, 2020, vol. 331, no. 3, pp. 135-144. In Rus.

2. Belov K.V., Lisenkov A.V., Ponomarev A.D., Gorbatenko N.S. Study of fluid filtration in a porous medium using physical and numerical modeling. Bulletin of the Tomsk Polytechnic University. Geo Assets Engineering, 2017, vol. 328, no. 8, pp. 64-74. In Rus.

3. Sokhoshko S.K. Accounting for changes in the filtration law when calculating the flow rate of a gas well. Bulletin of the Tomsk Polytechnic University. Geo Assets Engineering, 2020, vol. 331, no. 12 pp. 77-83. In Rus.

4. Halkarni S.S., Sridharan A., Prabhu S.V. Experimental investigation on effect of random packing with uniform sized spheres inside concentric tube heat exchangers on heat transfer coefficient and using water as working medium. International Journal of Thermal Sciences, 2018, vol. 133, pp. 341-356.

5. Yurkevich N.V., Yurkevich N.V., Gureyev V.N., Mazov N.A. Problems of controlling water filtration in hydraulic structures in permafrost regions. Bulletin of the Tomsk Polytechnic University. Geo Assets Engineering, 2020, vol. 331, no. 4, pp. 126-138. In Rus.

6. Bogoyavlenskii R.G. Gidrodinamika i teploobmen v vysokotemperaturnykh yadernykh reaktorakh s sharovymi tvelami [Hydrodynamics and heat exchange in the high-temperature nuclear reactors with pebble bed]. Moscow, Atomizdat Publ., 1978. 112 p.

7. Sorokin V.V. Gidravlika $i$ teploobmen sharovykh zasypok $v$ usloviyakh aktivnoy zony vodo-vodyanykh yadernykh reaktorov s mikrotvelami [Hydraulics and heat exchange of ball fillings in conditions of the active zone of water-water nuclear reactors with micro heat highlighting element]. Minsk, Belarus nauka Publ., 2010. $193 \mathrm{p}$.

8. Borozdin A.V., Varava A.N., Dedov A.V., Komov A.T., Malahovskiy S.A., Smorchkova Yu.V. Eksperimentalnoe issledovanie i chislennoe modelirovanie gidrodinamiki $\mathrm{i}$ teploobmena $\mathrm{v}$ sharovykh zasypkakh [Experimental study and numerical simulation of fluid flow and heat transfer in the pebble bed]. Teplovye protsessy v tekhnike, 2015, vol. 7, no. 7, pp. 295-300.

9. Avdeev A.A., Zudin Yu.B., Balunov B.F., Rybin R.A. An experimental investigation of heat transfer in a pebble bed. High Temperature, 2009, vol. 47, no. 5, pp. 692-700.

10. Lerou J.J., Froment G.F. Velocity, temperature and conversion profiles in fixed bed catalytic reactors. Chemical Engineering Science, 1977, vol. 32, Iss. 8, pp. 853-861.

11. Vafai K. Convective flow and heat transfer in variable-porosity media. Journal of Fluid Mechanics, 1984, vol. 147, pp. 233-259.

12. Poulikakos D., Renken K. Forced convection in a channel filled with porous medium, including the effects of flow inertia, variable porosity, and Brinkman friction. Journal of Heat Transfer, 1987, vol. 109, pp. 880-888.

13. Goldshtik M.A. Protsessy perenosa v zernistom sloe [Transfer processes in the granular layer]. Novosibirsk, SO RAN Publ., 2005. 358 p.

14. Li C.-H., Finlayson B.A. Heat transfer in packed beds - a reevaluation. Chemical Engineering Science, 1977, vol. 32 (9), pp. 1055-1066.

15. Vafai K., Tien C.L. Boundary and inertia effects on flow and heat transfer in porous media. International Journal of Heat and Mass Transfer, 1981, vol. 24, Iss. 2, pp. 195-203. 
16. Lee D.Y., Vafai K. Analytical characterization and conceptual assessment of solid and fluid temperature differentials in porous media. International Journal of Heat and Mass Transfer, 1999 , vol. 42, pp. 423-435.

17. Teplitskii Y.S. Heat exchange in a tube filled with granular bed. Journal of Engineering Physics and Thermophysics, 2004, vol. 77 (1), pp. 103-110.

18. Siraev R.R. Filtratsiya zhidkosti v poristoy srede Fortsgeymera s prostranstvenno neodnorodnymi poristostyu i pronitsaemostyu [Filtering of fluid in the porous medium of Fortzimemer with spatially inhomogeneous porosity and permeability]. Vychislitelnaya mekhanika sploshnykh sred, 2019, vol. 12, no. 3, pp. 281-292.

19. Yagi S., Kunii D. Studies on heat transfer in packed beds. International Development in Heat Transfer, 1961, Pt. IV, pp. 750-759.

20. Storck A., Coeuret F. Mass transfer between a flowing liquid and a wall or an immersed surface in fixed and fluidized beds. Chemical Engineering Journal, 1980, vol. 20, pp. 149-156.

21. Kays W.M., London A.L. Compact Heat Exchangers. New York, McGraw Hill Publ., 1984. 352 p.

22. Wakao N., Kaguei S., Funazkri T. Effect of fluid dispersion coefficients on particle-to-fluid heat transfer coefficients in packed beds: Correlation of Nusselt numbers. Chemical Engineering Science, 1979, vol. 34 (3), pp. 325-336.

23. Dekhtyar' R.A., Sikovsky D.Ph., Gorine A.V., Mukhin V.A. Heat transfer in a packed bed at moderate values of the Reynolds number. High Temperature, 2002, vol. 40, no. 5, pp. 693-700.
24. Dekhtyar R.A., Ovchinnikov V.V. An experimental study of hydrodynamics and heat transfer at fluid filtration through a porous medium. Journal of Physics: Conference Series, 2019, vol. 1359, 012122 .

25. Melanson M.M., Dixon A.G. Solid conduction in low dt/dp beds of spheres, pellets and rings. International Journal of Heat and Mass Transfer, 1985, vol. 28, pp. 383-394.

26. Wang B-X., Du J-H. Forced convection heat transfer in a vertical annulus filled with porous media. International Journal of Heat and Mass Transfer, 1993, vol. 36, no. 17, pp. 4207-4213.

27. Verschoor H., Schuit G.C.A. Heat transfer to fluids flowing through a bed of granular solids. Applied Scientific Research, 1951 A2, pp. 97-119.

28. Nilles M., Martin H. Heat transfer at the wall of packed beds. Proceedings 9th International Heat Transfer Conference. New York, Hemisphere Publishing, 1990. pp. 255-261.

29. Aerov M.E., Todes O.M. Gidravlicheskie i teplove osnovy raboty apparatov so statsionarnym zernistym sloem [Hydraulic and thermal bases of apparatuses with stationary grain layers]. Leningrad, Chimiya Publ., 1968. 510 p.

30. Yagi S., Wakao N. Heat and mass transfer from wall to fluid in packed beds. American Institute of Chemical Engineers Journal, 1959, vol. 5, no. 1, pp. 79-85.

Received: 2 August 2021.

\section{Information about the authors}

Ruslan A. Dekhtyar, Cand. Sc., senior researcher, S.S. Kutateladze Institute of Thermophysics SB RAS. 